\title{
Surgery of Parathyroid
}

\author{
Sai Krishna Vittal $\cdot$ Sai Vishnupriya V. $\cdot$ Sucharitha V. $\cdot$ S. Vittal
}

Received: 24 February 2009 / Accepted: 11 November 2009

(C) Association of Surgeons of India 2009

\begin{abstract}
Surgery of parathyroid revolves around the management of hyperparathyroidism (HPT). In most cases, occurrence is sporadic rather than familial, and $80-85 \%$ of cases of sporadic primary HPT are caused by a solitary parathyroid adenoma. The diagnosis is made by hypercalcaemia with an inappropriately elevated parathyroid hormone (PTH) level and a 24-hour urine calcium excretion level that is normal or high. Improved assay for PTH has led to earlier detection of HPT and has been responsible for the apparent increase in the prevalence of the disorder. An improvement in preoperative localisation studies as well as the development of a rapid intraoperative PTH assay has changed the approach to parathyroid surgery in the last two decades. This article provides a brief overview of management of primary HPT.
\end{abstract}

Keywords Primary hyperparathyroidism . Hypercalcaemia $\cdot$ Sestamibi scan $\cdot$ Parathyroidectomy

S. K. Vittal · Sai Vishnupriya V. · Sucharitha V. · S. Vittal Department of Surgery

Vittals Institute of Endocrine and Laparoscopic Surgery, 32 Kilpauk Garden Road, Kilpauk, Chennai - 600010, India

S. Vittal $(\bowtie)$

E-mail: svittal@vsnl.com

\section{History}

The parathyroid glands were first identified in 1850 in an Indian Rhinoceros in a London zoo by Richard Owen, then Professor of Anatomy at the Hunterian Museum of the Royal College of Surgeons of England [1]. His work published in 1862 went unnoticed for several years. Credit for the recognition of the parathyroid glands in humans went to Ivar Sandstrom a medical student at Uppsala University in Sweden who named them "glandulae parathyroidae". The first parathyroidectomy for primary HPT was performed by Mandl in 1925 [2].

\section{Surgical anatomy and physiological aspects}

A thorough understanding of the anatomy of parathyroid glands is essential before embarking on parathyroid exploration. The parathyroid glands usually four in number arise from the endoderm of the third and fourth pharyngeal pouches. The superior parathyroids arise from the fourth pharyngeal pouch and are usually present superior to the inferior thyroid artery and dorsal to the recurrent laryngeal nerve. The inferior parathyroid glands arise from the third pharyngeal pouch and descend with the thymus into the lower part of the neck [3]. Due to this longer migration, they may be found at any position in the neck and the mediastinum, although the majority lie within $1-2 \mathrm{~cm}$ of the lower pole of the thyroid gland. The inferior parathyroids usually lie inferior to the inferior thyroid artery and ventral to the recurrent laryngeal nerve. Although most people have four parathyroid glands, a range from 2 to 6 have been described.

Parathyroid hormone (PTH) is an intact 84 amino acid peptide with amino and carboxyl terminals that is secreted in response to a fall in plasma ionised calcium concentration. The circulating PTH which has a half-life of 
about 5 minutes in patients with normal renal function, is initially cleaved in the liver, yielding an inactive C-terminal fragment, which is ultimately cleared by the kidney [4]. The N-terminal fragment is the part of the peptide that is responsible for the biological activity of PTH on peripheral tissues. PTH interacts with vitamin D and its metabolites in regulating calcium absorption and secretion. PTH has direct effects that promote reabsorption of calcium from renal tubules and bone. PTH has indirect effects, mediated by increasing renal conversion of 25-hydroxycholecaciferol to more potent hormone 1,25 dihydroxycholecalciferol which results in increased calcium absorption from food.

The surgery of parathyroids revolves around the management of primary HPT and hence the management of primary HPT is discussed in detail below.

\section{Primary hyperparathyroidism}

Primary HPT is the detection of hypercalcaemia in the presence of inappropriately elevated circulating PTH levels. Primary HPT is the most common cause of hypercalcaemia in unselected non-hospitalised patients. It is the second most common cause of hypercalcaemia (after malignancy) in hospitalised patients.

The exact cause of sporadic primary HPT is unknown and is likely multifactorial, with environmental and genetic causes. It is associated with a history of radiation exposure $[5,6]$ as well as with prolonged lithium use [7, 8]. Genetic associations in sporadic primary HPT include over expression of the PRAD1 oncogene (encoding cyclin D1) and an inactivating mutation of the multiple endocrine neoplasia type 1 (MEN1) tumour-suppressor gene (encoding menin) [9]. The MEN1 gene is also associated with familial HPT, as are RET (associated with MEN2), HRPT2 (encoding parafibromin, associated with HPT-jaw tumour syndrome), and the CASR gene (encoding the calcium-sensing receptor, associated with neonatal primary HPT).

\section{Incidence}

There is, however, a higher prevalence with increasing age, especially in females. In the age group below 40, the incidence of HPT is about 10 cases per 100,000 populations. In those over the age of 60 years, there is a steep rise in incidence to about $91 / 100,000$ in men and $188 / 100,000$ in women. The incidence is highest in the third to fifth decade.

\section{Pathology}

Primary HPT may be sporadic or familial. In most cases (80-85\%), sporadic primary HPT is caused by a solitary parathyroid adenoma, with the remainder of cases due to double adenomas (about 4\%), multiple-gland hyperplasia $(10-15 \%)$ and parathyroid carcinoma $(<1 \%)$. Familial syndromes associated with primary HPT include MEN1 and MEN2, non-MEN, familial HPT, HPT-jaw tumour syndrome, and familial neonatal HPT. These familial syndromes are associated with (usually asymmetric) multiple-gland hyperplasia. In addition, non-MEN familial HPT and HPT-jaw tumour syndrome are associated with an increased risk of parathyroid cancer.

Hyperplasia of all four parathyroids is typical of secondary HPT. However, hyperplasia is responsible for the primary disease in about $10 \%$ of cases and may affect two or more glands. The chief cells are frequently involved. In contrast to an adenomatous gland, the hyperplastic parathyroid shows no rim of normal tissue and the overall appearance is uniform. Nodular hyperplasia is a feature strongly associated with MEN syndrome.

Carcinoma parathyroid is a rare cause of HPT accounting for $<2 \%$ of all cases of primary HPT. Most cases occur in the fourth to sixth decade of life and in contrast to the female predominance observed for adenomas, there are no sex differences in the incidence of carcinomas [10]. A visible and palpable lump in the thyroid region along with hypercalcaemia should alert the surgeon to the possibility of diagnosis of carcinoma of the parathyroid. The definitive diagnosis is only made postoperatively from histopathology. Typically, the carcinoma is firm to hard, grey in colour and adherent to the adjacent tissue. The distant metastases to the liver and bone is a late feature and indicate terminal disease.

Clinical features

\begin{tabular}{ll}
\hline Fatigue & Nephrolithiasis/renal failure \\
Anorexia & Hypertension/heart blocks \\
Weight loss/vomiting & Osteopaenia \\
Epigastric pain & Bone fracture \\
Polyuria/nocturia & Gout/pseudogout \\
Bone pain & Pancreatitis \\
Back pain & Duodenal ulcer \\
Constipation & Gastric ulcer \\
Depression & Dementia \\
\hline
\end{tabular}

\section{Diagnosis of primary HPT}

- Increase in serum calcium

- Increase in PTH. There is an inappropriate increase in serum calcium with relation to serum PTH.

- Twenty-four hours urinary calcium is done to exclude familial hypocalciuric HPT (FHH). In patients with $\mathrm{FHH}$, there is a mutation in extracellular calcium sensor. This causes increased renal tubular reabsorption of calcium, which results in urinary calcium levels $<150$ $\mathrm{mg} / 24$ hours. The most accurate way to diagnose FHH 
is by calculating the calcium-to-creatinine clearance ratio. It is extremely important to identify patients with FHH because they remain asymptomatic throughout life and therefore, do not require any surgical intervention. A urinary calcium excretion of $400 \mathrm{mg} / 24$ hours or higher is generally considered an indication for surgical exploration.

Normocalcaemia does not exclude HPT. Complementary tests that support the diagnosis and identify patients who have associated deleterious effects of HPT are as follows.

- Plasma phosphate: As more phosphate is lost in urine, serum phosphate levels falls.

- Plasma creatinine: Indicates renal function.

- Alkaline phosphatase: Indicator of bone disease.

- Chloride: Phosphate ratio of $>33$ indicative of primary HPT

\section{Normocalcaemic hyperparathyroidism}

There is a small subset of patients with primary HPT who present with normal or intermittently elevated calcium levels. The exact biochemical mechanisms of normocalcaemic primary HPT remain unknown. It has been postulated by some that normocalcaemic variant of primary HPT represents an early or preclinical phase that progresses to typical hypercalcaemic primary HPT $[11,12]$. The majority of patients with normocalcemic primary HPT present with renal calculi and hypercalciuria.

\section{Surgical intervention}

Primary HPT is essentially a surgical disease. Surgery achieves normocalcaemia in about $95 \%$ of cases. Surgery is indicated in all patients with symptomatic primary HPT and in acute primary HPT (parathyroid crisis). Patients with primary HPT presenting with osteitis fibrosa cystica (brown tumour) are nowadays uncommon (Figs 1 and 2). A change in clinical profile and better diagnostic assays have resulted in more cases of asymptomatic HPT. There has been a paradigm shift in the threshold for surgical intervention in asymptomatic patients. In 1990, the National Institutes of Health (NIH) convened a consensus conference on the management of asymptomatic primary HPT [13]. These guidelines were then revised in 2002, at the workshop on asymptomatic primary HPT [14].

What are the indications for surgery in patients with asymptomatic HPT?

- Serum calcium $>1.0 \mathrm{mg} / \mathrm{dl}(0.25 \mathrm{mM})$ above the reference range

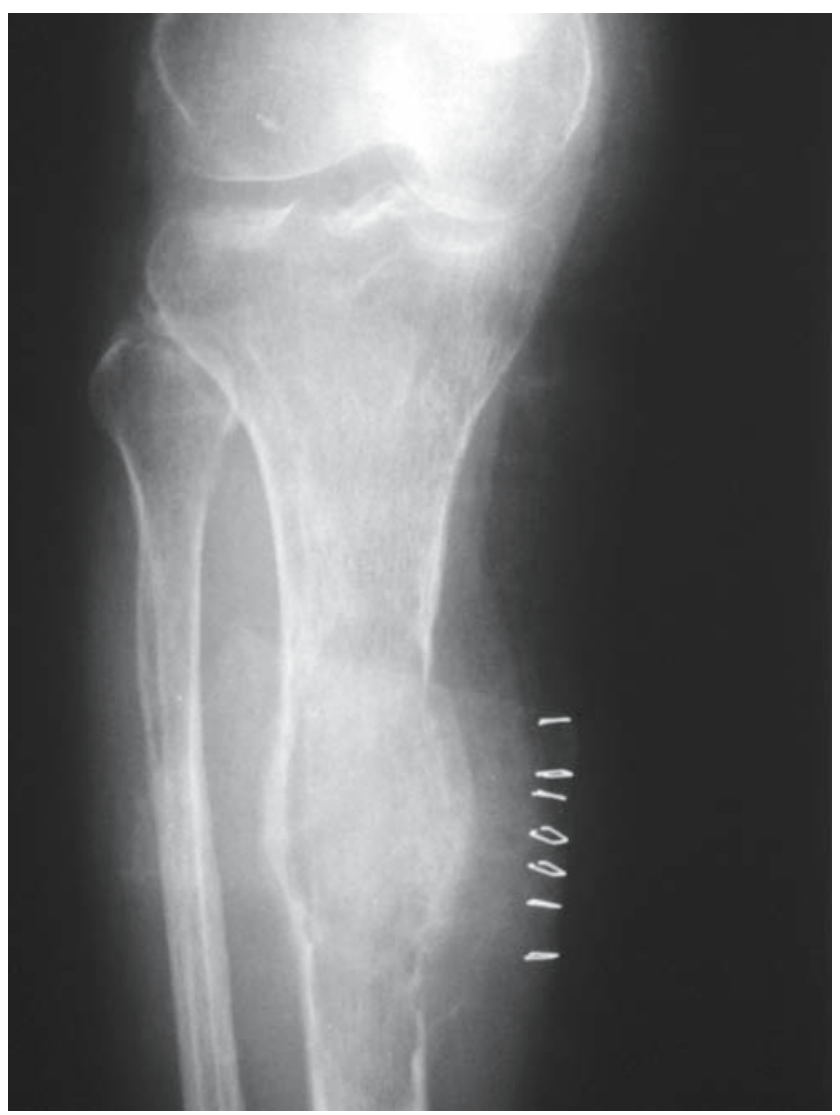

Fig. 1 X-ray showing Brown tumour of tibia and skin clips indicating biopsy site

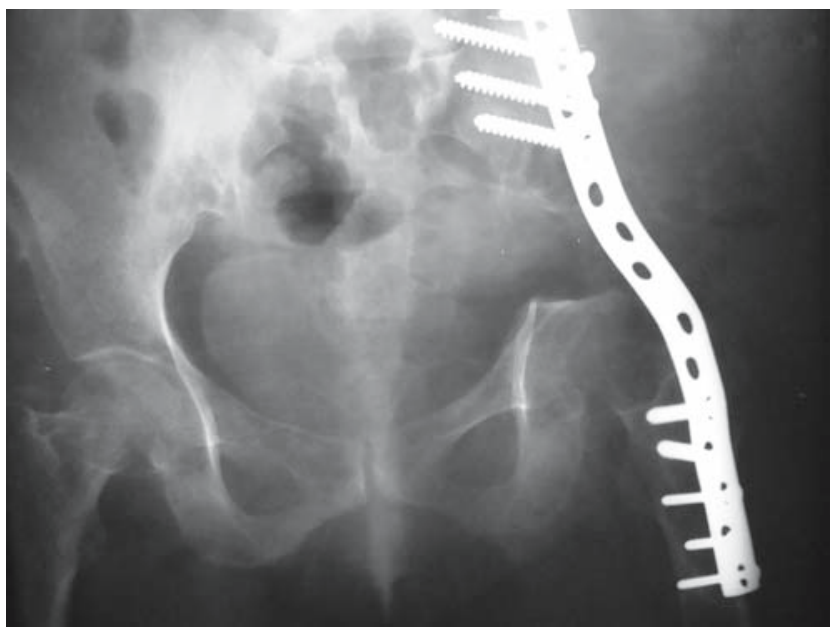

Fig. 2 X-ray pelvis showing left internal haemipelvectomy for "giant cell tumour of ilium" in a patient who was later discovered to have a parathyroid carcinoma

- Twenty-four hours urinary calcium excretion $>400 \mathrm{mg}$ (10 mmol)

- Creatinine clearance reduced by $30 \%$ compared with age-matched control subjects

- Forearm, lumbar spine, or hip T score reduced by $>2.5$ $\mathrm{SD}$ on bone mineral density scan 
- Patients younger than age 50 years

- Patients for whom medical surveillance is either not desirable or possible.

\section{Preoperative localisation}

The rationale for locating the abnormal parathyroid before surgery is that the glands can be notoriously unpredictable in their location. An experienced surgeon should be able to locate the parathyroid in about $95 \%$ of cases. However at times the localisation of parathyroid becomes difficult especially when the patient has had previous neck surgery, or in ectopic positions of parathyroids. Although ultrasound scan of the neck, CT scan and MRI scan and selective venous sampling have been used, the localising test of choice is Technetium-99m $\left({ }^{99 m} \mathrm{Tc}\right)$ sestamibi scan (Fig. 3). Thallium Technetium which was widely used previously has now been largely replaced by sestamibi scan. The fortuitous discovery by Coakley et al. [15] that ${ }^{99 \mathrm{~m}} \mathrm{Tc}$ sestamibi concentrates in abnormal parathyroid glands has revolutionised the practice of parathyroid surgery.

Sestamibi is an isonitrile compound, which is labeled with ${ }^{99 m} \mathrm{Tc}$ to form a cationic complex. Sestamibi

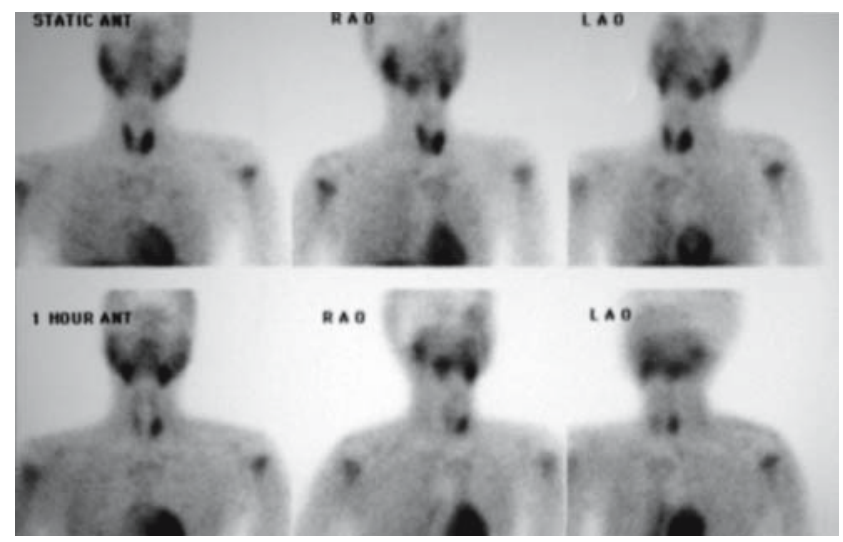

Fig. 3 Sestamibi scan indicating enlarged left lower parathyroid

accumulates in the mitochondria of cells and therefore a tissue with large number of mitochondria may take up sestamibi more avidly. It has been shown that parathyroid adenomas have a large number of mitochondria in their cells and it is therefore possible that it would be taken up more avidly in adenomatous tissue than the surrounding thyroid and following uptake a slower release would occur from the parathyroid cell. Despite the high sensitivity rate for parathyroid disorders especially parathyroid adenomas, an important limitation of this technique is false positive findings. The common cause of false positive are multinodular goitre, hashimotos thyroiditis, thyroid adenomas and thyroid carcinoma. Sestamibi scan is now considered mandatory before operative intervention in persistent or recurrent HPT.

\section{Bilateral neck exploration}

The surgical gold standard is a bilateral neck exploration with identification of all four parathyroid glands and resection of the abnormal gland(s). This procedure has a success rate of about $95-98 \%$ for curing primary HPT, with minimal morbidity, mortality close to zero and excellent cosmetic results [16].

\section{Exposure and technique of exploration}

Incision and exposure is similar to that of thyroid surgery. Meticulous haemostasis is crucial. The middle thyroid vein should be divided to allow full mobilisation of thyroid lobe to search for parathyroids. If need be, the fascia on the posteromedial aspect of thyroid is incised to increase the area of exposure. Upper parathyroids are located within $2 \mathrm{~cm}$ radius of the site of division of inferior thyroid artery. The inferior gland is less constant in position. It is found in $80 \%$ of cases within $2 \mathrm{~cm}$ radius of inferior pole of thyroid. If not localised the following sites should be searched for ectopic parathyroid.

- Thyrothymic ligament

- Tracheooesophageal groove and behind the oesophagus

- Intrathyroidal

- Carotid sheath, so opening the sheath may reveal the parathyroids

- Transcervical thymectomy may be necessary especially in MEN

- Median sternotomy may be used as the last resort.

In case of single gland adenoma, surgical removal gives a complete cure (Fig. 4). In case of multiglandular hyperplasia, the strategy is to do a subtotal parathyroidectomy where three glands are removed and a small portion of the fourth gland $(40-60 \mathrm{~g})$ is left behind

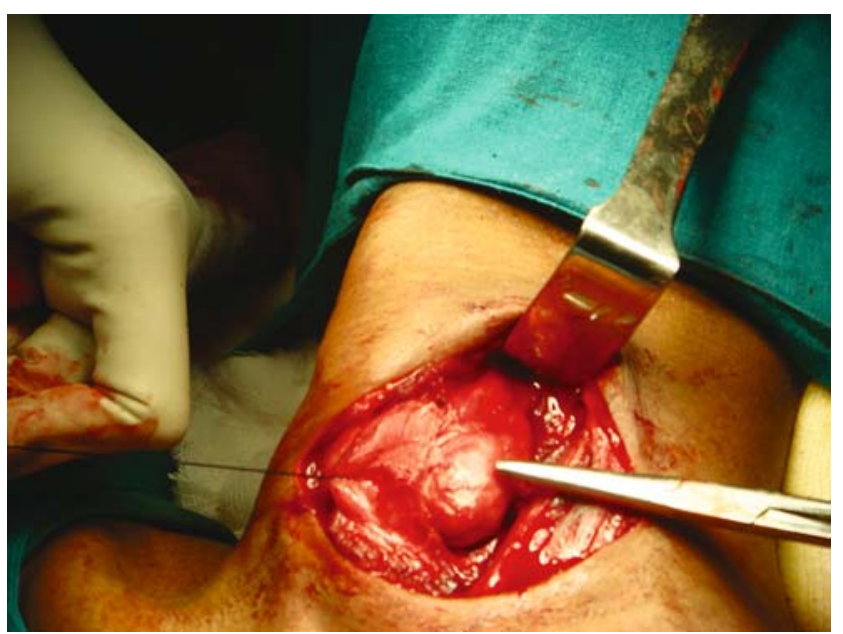

Fig. 4 Operative photo showing removal of enlarged left lower parathyroid which was proven histopathologically to be a parathyroid adenoma 
with intact blood supply. The alternative approach would be to do total parathyroidectomy and autotransplantation of parathyroid tissue in sternocleidomastoid muscle or the non-dominant forearm (brachioradialis). In case of parathyroid carcinoma, enbloc resection of parathyroid with ipsilateral haemithyroidectomy and nodal dissection is recommended.

All the tissues removed should be confirmed by frozen section. Patient undergoing either subtotal or total parathyroidectomy with autotransplantation are at risk of developing permanent HPT. To avoid this complication it is recommended that parathyroid tissue routinely be saved and cryopreserved for patients with multiglandular disease.

\section{Unilateral neck exploration}

Given the fact that $80-85 \%$ of patients will have only a solitary adenoma, a bilateral neck exploration subjects $15-20 \%$ of patients to unnecessarily extensive surgery, with the attendant risks of recurrent laryngeal nerve injury and postoperative hypocalcaemia. This has prompted some surgeons to perform a unilateral neck exploration [17]. This change in practice has been made possible because of an improvement in preoperative localisation studies and the development of intraoperative PTH monitoring. Unilateral exploration relies not only on the ability to localise an adenoma preoperatively but also on careful patient selection. Patients with mutiglandular disease, MEN related hyperplasia and renal disease are not suitable for this approach.

\section{Minimally invasive parathyroid surgery}

Minimally invasive radio-guided parathyroidectomy

Patients with primary HPT undergo localisation of their tumours with sestambi scan. The procedure uses an intraoperative gamma-probe to direct the dissection according to the level of radioactivity. This probe helps the operator to focus directly on to the tumour location. It can prove useful in persistent or recurrent HPT. Good results have been obtained from this technique [18].

Minimally invasive endoscopic parathyroidectomy

This technique was first described by Gagner $[19,20]$. Preoperative localisation with a sestamibi scan is necessary. Multiple ports are used for endoscopic dissection, the camera and gas insufflation. The gland is then retrieved via the largest incision. A quick PTH assay is performed after resection. Bilateral parathyroid exploration is possible.
Minimally invasive video-assisted parathyroidectomy

This technique requires no trocars or gas insufflations [21]. This technique gains access to the neck through a small midline incision made at the suprasternal notch. Once inside the neck the entire procedure is performed by blunt dissection endoscopically using small reusable surgical instruments. Preoperative localisation with a sestamibi scan is essential and usually combined with intraopertive PTH measurement. This technique also permits a bilateral exploration.

\section{Intraoperative PTH measurement}

The introduction of intraoperative PTH assay has been an important advance in the development of unilateral neck exploration. Intraoperative PTH is measured using quick assay method and a $50 \%$ reduction in baseline value of PTH after 10 minutes of excision of adenoma predicts postoperative normocalcaemia [22]. It should however be noted that this technique is not fool proof and one must be aware that both false positive and false negative results have been reported.

\section{Secondary and tertiary HPT}

Secondary HPT is the increased production of PTH in response to prolonged hypocalcaemia and is associated with hyperplasia of all parathyroid tissue. It is usually seen in patients with chronic renal failure and vitamin $\mathrm{D}$ deficiency. Most of them can be managed by medical therapy. Surgery may be indicated when there is uncontrollable hypercalcaemia, hyperphosphataemia, high levels of PTH $(>500 \mathrm{pg} / \mathrm{ml})$, bone erosions and osteitis fibrosa. Surgical options include either subtotal parathyroidectomy or total parathyroidectomy with autotransplantation. In a small proportion of cases of secondary HPT, continuous stimulation of the parathyroids results in adenoma formation and autonomous PTH secretion independently of calcium level. This is known as tertiary HPT. It is most frequently seen in patients after kidney transplantation.

Recently, drugs that enhance the sensitivity of calciumsensing receptor (calcimimetics) [23] are being developed and in the future may offer an alternative to surgery in HPT. Routine serum calcium estimation may help in recognising many asymptomatic as well as symptomatic cases of HPT [24].

\section{Conclusion}

- Primary HPT can be definitely diagnosed with elevated PTH in hypercalcaemic patients without hypocalciuria.

- Surgery should now be considered early in the natural history of the primary HPT, even in asymptomatic patients. 
- Unilateral neck exploration are acceptable with preoperative localisation and peroperative PTH monitoring in appropriate cases.

- Bilateral neck exploration remains a safe approach with an excellent success rate and is still regarded as gold standard.

- Whether unilateral exploration is superior to bilateral approach in success rate, complication rate or cost effectiveness remains to be proved by more prospective randomised studies.

\section{References}

1. Modorai B, Sawyer A, Ellis H (2004) The Glands of Owen. J R Soc Med 97:494-495

2. Mandl F (1926) Klinisches und Experimenteles zur Frage der lakalisierten und generalisiereten Osteitis Fibrosa. Arch Klin Chir 143:1

3. Moore MA, Owen JJ (1967) Experimental studies on the development of the thymus. J Exp Med 126(4):715-726

4. Libutti SK, Alexander HR, Bartlett DL, et al. (1999) Kinetic analysis of rapid intraoperative parathyroid hormone assay in patients during operation for hyperparathyroidism. Surgery 126(6):1145-1150; discussion 1150-1151

5. Beard CM, Heath H, O'Fallon WM, Anderson JA, Earle JD, Melton LJ (1989) Therapeutic radiation and hyperparathyroidism. A case-control study in Rochester. Minn Arch Intern Med 149(8):1887-1890

6. Cohen J, Gierlowski TC, Schneider AB (1990) A prospective study of hyperparathyroidism in individuals exposed to radiation in childhood. JAMA 264(5):581-584

7. Garfinkel PE, Ezrin C, Stancer HC (1973) Hypothyroidism and hyperparathyroidism associated with lithium. Lancet 2(7824):331-332

8. McIntosh WB, Horn EH, Mathieson LM, Sumner D (1987) The prevalence, mechanism and clinical significance of lithium-induced hypercalcaemia. Med Lab Sci 44(2): 115-118

9 Arnold A, Shattuck TM, Mallya SM, et al. (2002) Molecular pathogenesis of primary hyperparathyroidism. J Bone Miner Res 17(Suppl 2):N30-N36

10 Shane E (1994) Parathyroid carcinoma. In The Parathyroids: Basic and Clinical Concepts. Bilezikian JP, Marcus R, Levine MA (Eds.), Raven, New York
11. Silverberg SJ, Bilezikian JP (2003) Incipient primary hyperparathyroidism: A "forme fruste" of an old disease. J Clin Endocrinol Metab 88(11):5348-5352

12. Carnaille BM, Pattou FN, Oudar C, et al. (1996) Parathyroid incidentalomas in normocalcemic patients during thyroid surgery. World J Surg 20(7):830-834; discussion 834

13. Proceedings of the NIH Consensus Development Conference on diagnosis and management of asymptomatic primary hyperparathyroidism; Bethesda, Maryland, October 29-31, 1990. Miner Res 1991;6(Suppl 2):S1-S166

14. Bilezikian JP, Potts JT Jr, Fuleihan Gel-H, et al. (2002) Summary statement from a workshop on asymptomatic primary hyperparathyroidism: A perspective for the 21st century. J Clin Endocrinol Metab 87(12):5353-5361

15. Coakley AJ, Kettle AG, Wells CP, et al. (1989) $\mathrm{T}_{\mathrm{cm}}$ sestamibi - a new agent for parathyroid imaging. Nucl Med Commun 10(11):791-794

16. Van Heerden JA, Grant CS (1991) Surgical treatment of primary hyperparathyroidism: An institutional perspective. World J Surg 15:688-692

17. Russel CF, Laird JD, Fergusson WR (1990) Scan-directed unilateral cervical exploration for parathyroid adenoma: A legitimate approach ? World J Surg 14:406-409

18. Goldstein RE, Blevins L, Delbeke D, et al. (2000) Effect of minimally invasive radioguided parathyroidectomy on efficacy, length of stay and costs in management of hyperparathyroidism. Ann Surg 231:732-742

19. Gagner M (1996) Endoscopic subtotal parathyroidectomy in patients with primary hyperparathyroidism. Br J Surg 83:875

20. Gagner M, Rubino F (2004) Endoscopic parathyroidectomy. In: Endocrine Surgery. Schwartz AE, Pertsemlidis D, Gagner M (Eds.), Marcel Dekker, New York 289-296

21. Miccoli P, Bendinelli C, Conte M (1998) Endoscopic parathyroidectomy by a gasless approach. J Laparoendosc Adv Surg Tech A 8:189-194

22. Udelsman R, Donovan PI, Sokoll LJ (2000) One hundred minimally invasive parathyroid explorations. Ann Surg 232: 331-339

23. Shoback DM, et al. (2001) An evaluation of the calcimimetic AMG 073 in patients with hypercalcemia and primary hyperparathyroidism. J Bone Miner Res 16:S303

24. Shukla S, Kaushal M, Shukla SK (2008) Primary hyperparathyroidism: retrospective 10-year study of 32 cases. Indian J Surg 70:169-174 